\title{
Modified Stacked Compact Microstrip Patch Antenna for Implantable Medical Devices
}

\author{
Dheeraj Bhardwaj \\ Assistant Professor, Department of Physics, \\ BIT-Mesra- Jaipur Campus, Jaipur, India \\ Sahil Tangri \\ Bachelor of Engineering-ECE, Department of Electronics \& Communication Engineering, \\ BIT-Mesra- Jaipur Campus, Jaipur, India \\ Komal Sharma \\ Reader, Department of Physics, \\ Swami Keshvanand Institute of Technology, Jaipur , India
}

\begin{abstract}
The purpose of this paper was to design an antenna for implantable medical devices. This miniature implantable antenna is simulated using Rogers's 6010 substrate having dielectricity of 10.2. A compact and miniature dual-band implantable antenna consists of an unsymmetrical spiral radiating stripe and a U-shaped radiating stripe is proposed for MICS and ISM band (2.4-2.48 GHz) applications. The length and width of the patch are optimized to attain the best performance. The modified antenna for medical implant resonates at two different frequencies, i.e. $2.42 \mathrm{GHz}$ and 4.2 GHz with efficiency of $94 \%$. The modified antenna is a dual- layered antenna having the superstrate layer of Rogers 6010 having dielectricity of $\mathbf{1 0 . 2}$ with two shorting PIN, which enhances the antenna results.
\end{abstract}

Keywords - ROGER'S SUBSTRATE, IMPLANTABLE MEDICAL DEVICE, SHORTING PIN TECHNIQUE

\section{INTRODUCTION}

Devices that can be implanted in a living body have always attracted significant scientific interest for many diagnostic and therapeutic applications. The demand for non-invasive surgical operations has made the use of Implantable Medical Devices (IMDs) as part of medical procedures. Because devices need to communicate wirelessly with the equipments which are present or are external to the human or any living body, the implanted antennas are required. The dimensions of traditional half-wavelength antennas at the MICS band are useless for implantable applications. Hence, miniaturization becomes the greatest challenges in implanted antenna design. PIFAs have been used to implantable antennas by research groups because of the advantages. The use of wireless technology in the field of medicine brings together medical information with seamless communication, providing limit- less opportunities for improving health care. Medical professionals are now able to diagnose, oversee patients vital functions in acute and long-term situations and monitor the effectiveness of treatment plans in a superior way through the use of wireless medical devices. It also offers patients and caregivers access to systems that can support them in managing their conditions more easily and safely, thus improving their quality of life.The microstrip patch antenna, known as printed resonating antenna is very popular for narrow-band microwave wireless links which require semi-hemispherical coverage. Due to the small size, light weight, low profile, ease of integration with active and passive devices and ability of mounting on planar, non-planar as well as rigid exteriors to form MIC's, they are extensively studied by the scientific community. Designers have always been looking for creative ways to improve the performance of these antennas. One method used in patch antenna design is to introduce shorting pins (from the patch to the ground plane) at various locations which leads into the Planar Inverted-F Antenna.

\section{METHODS}

A. FINITE ELEMENT METHOD (FEM) 
The finite element method (FEM) is a numerical technique used for the finding approximated solutions to boundary valued problems for the partial differential equations. It is mostly referred to as the finite element analysis (FEA). FEM subdivides a larger problem into smaller, simpler parts, called the finite elements. The simple equations that model these finite elements are assembled on to a larger system of equations that models the entire problem. FEM is technology which uses variational methods from the help of calculus of variations to approximate all the solution by minimizing the associated error function. It is one of the powerful commercially available tools used for antenna designing, and for designing of complex RF electronic circuit elements which includes filters, transmission lines, and packaging. This is a procedure where the structures are to be subdivided into many smaller subsections called finite elements. The finite elements used are tetrahedral, and the entire collection of tetrahedral is called a mesh. A solution will always be found for the fields within the finite elements, and these fields are highly interrelated so that Maxwell's equations are made satisfied across all the inter-element boundaries. So one can achieve the field solution for the entire and the original structure. Once the field solution has been found, the generalized S-matrix solution can be determined.

\section{B. COAXIAL PROBE FEED TECHNIQUE}

The inner conductor of the SMA connector extends through the dielectric and is soldered to the radiating patch, while the outer conductor is connected to the ground plane as shown in Fig.1.

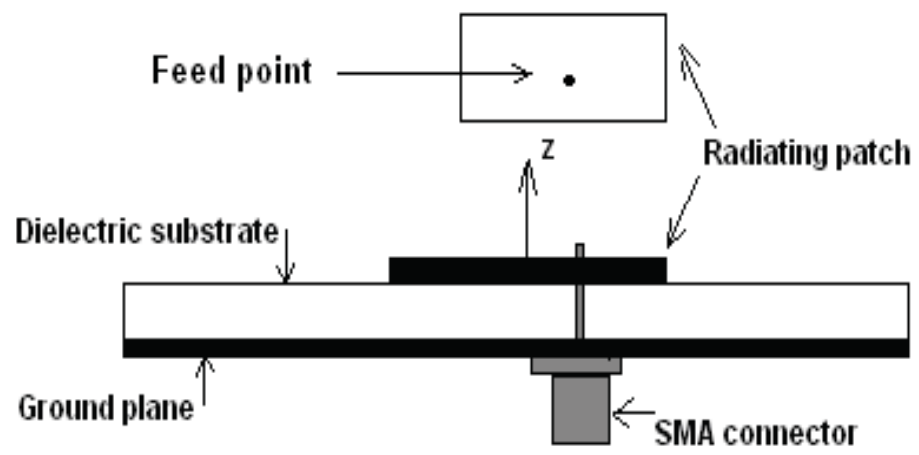

Fig. 1. Coaxial Probe feed technique

Microstrip antennas can be fed from underneath via a probe as shown in the figure 1 . The inner conductor of the coaxial connector extends through the dielectric and is soldered on to the radiating patch, while the outer conductor is connected to the ground plane. The only advantage of Probe feeding scheme is that the feed can be placed at any desired location inside the patch to match with its input impedance. The probe feed method is easy to fabricate and has low spurious radiation. The Coupling of power on to the patch antenna through the probe feed technique is quite simple, cheap, and effective way.

\section{ANTENNA DESIGN}

In the present work, microstrip patch antenna is taken in to consideration having patch dimensions $(17 * 16) \mathrm{mm}$ as shown in Fig. 2. The RF power is fed directly to the radiating patch using a using coaxial probe feed technique. It is situated in the XY-plane over an infinite ground plane with substrate thickness $\mathrm{h}=0.5 \mathrm{~mm}\left(\mathrm{~h}<<\lambda_{0}\right)$, substrate dielectric constant $\left(\varepsilon_{\mathrm{r}}=10.2\right)$, loss tangent $(\tan \delta=0.0023)$. 


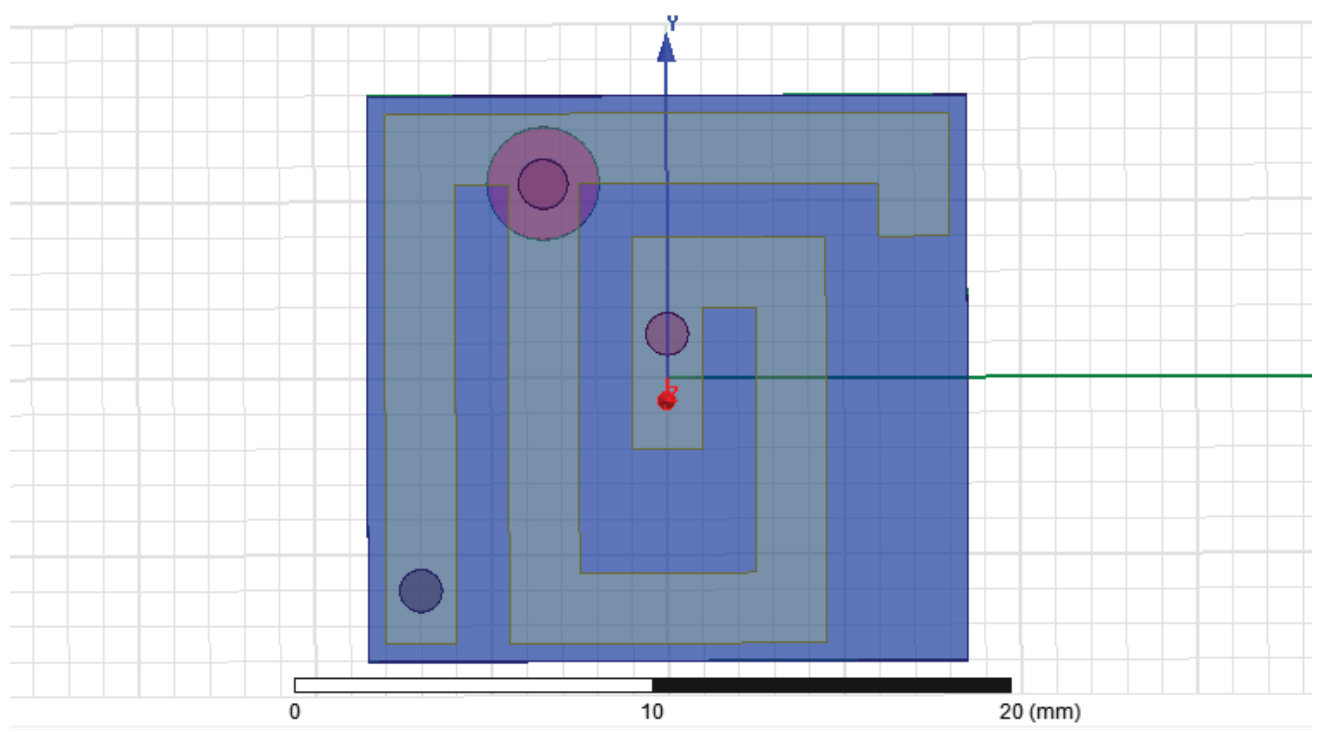

Fig. 2. Geometry of the proposed dual-band implantable antenna

The top view and side view of the proposed stacked dual-band implantable antenna is shown in Fig. 2 and Fig. 3 respectively. It consists of one substrate and one superstrate layer with a spiral radiating stripe and U-shaped radiating stripe. The spiral stripe can meander the current path, thus it can obtain a reduced size than conventional microstrip antenna for MICS band. The U-shaped radiating stripe is designed to work in ISM band. The radiator merging a spiral radiating stripe and a U-shaped radiating stripe has a compact structure, which was sandwiched between substrate and superstrate dielectric layers of Roger's 6010.

The addition of superstrate layer or stacking on the top layer of patch has several advantages. It is a miniaturization technique and hence vertically stacking of two radiating patches reduces antenna size by increasing (nearly doubling) the length of the current flow path. I have used one more technique i.e. addition of two shorting pin to enhancement of the performance of the antenna parameters.

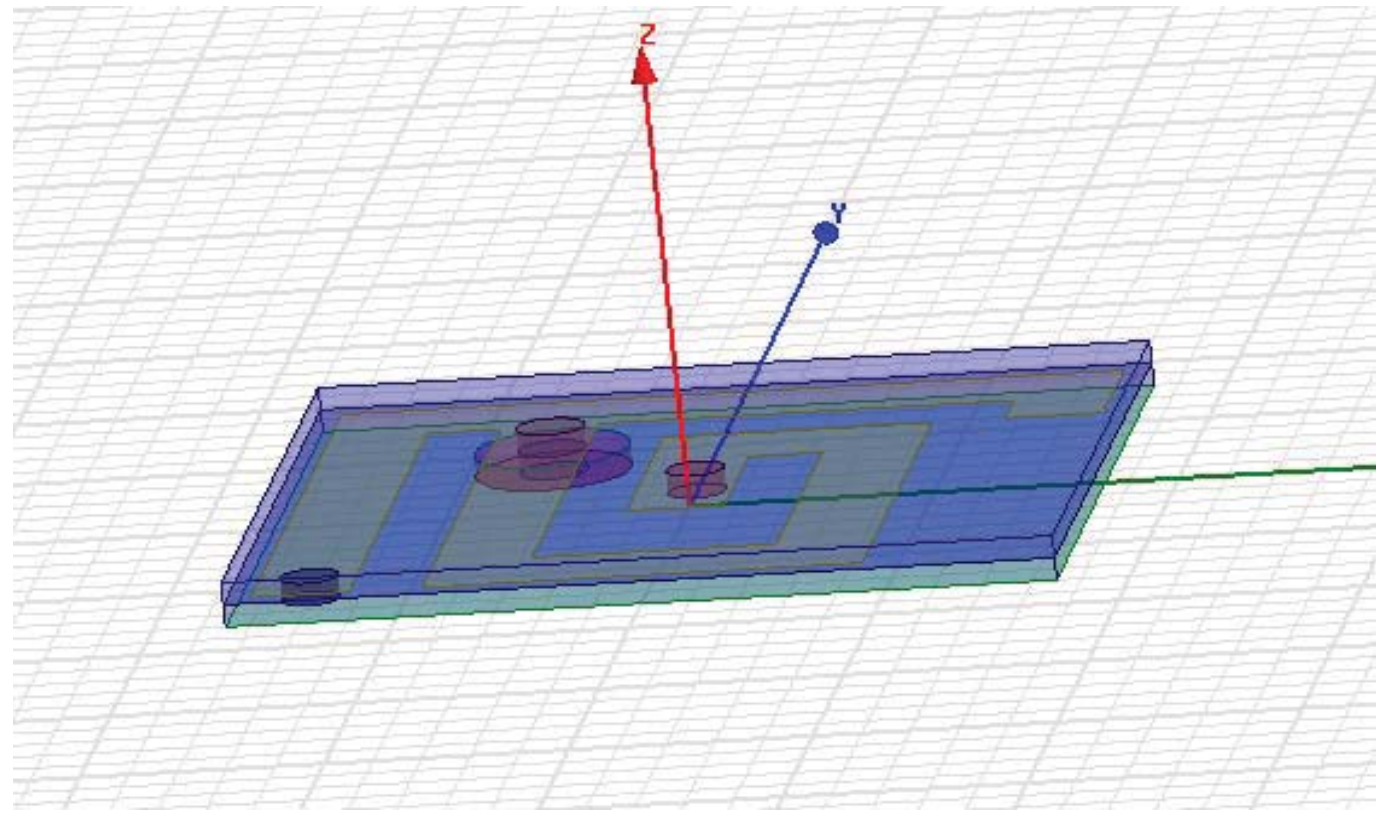

Fig. 3. Side view of the proposed dual-band implantable antenna 


\section{RESULTS AND ANALYSIS}

The designed implantable antenna for MICS band has been analysed and studied carefully. Fig 4 shows the return loss for the implantable antenna without the superstrate layer. To optimize the gain, directivity, radiation patterns over the operating range and to further increase the bandwidth, the superstrate layer was added.

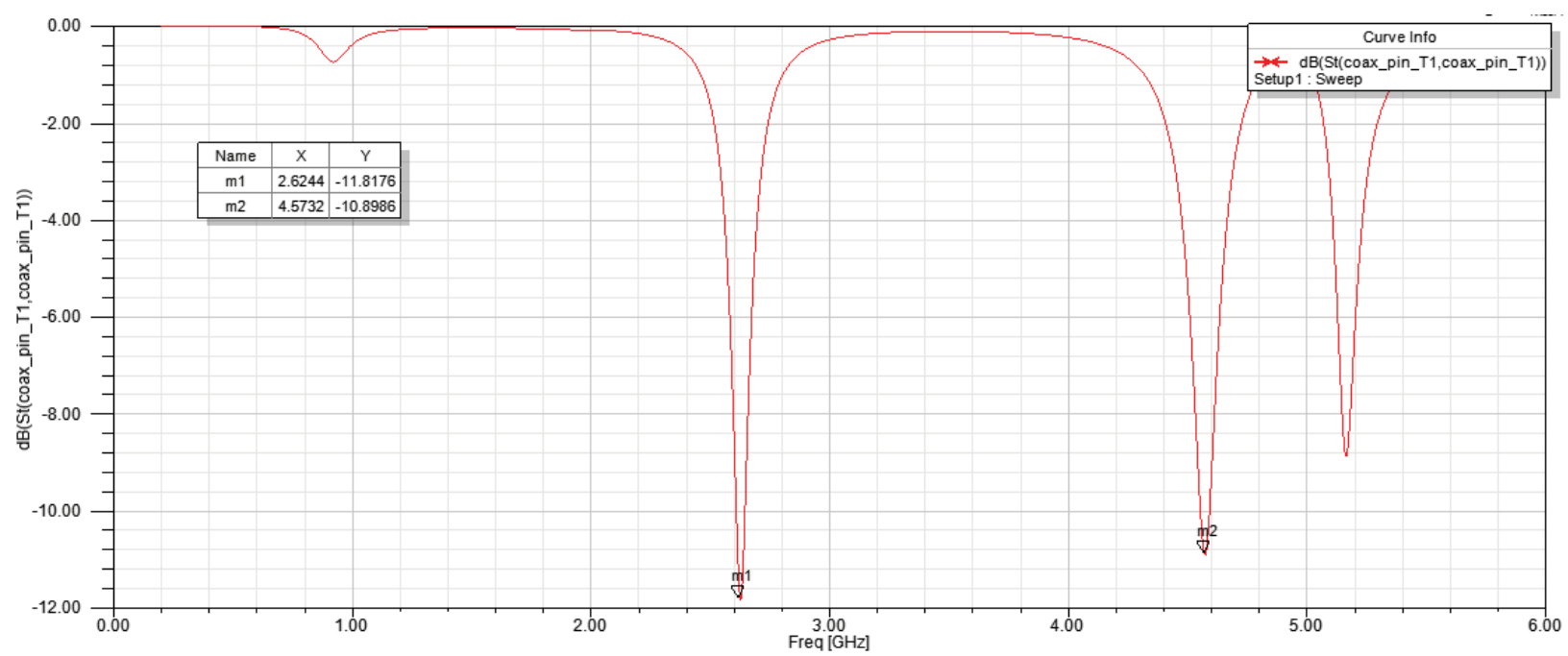

Fig. 4. Variation of return loss with frequency for implantable antenna (without superstrate layer)

The designed antenna having $\mathrm{S}_{11}$ parameter to be $-11.8176 \mathrm{~dB}$ and $-10.8986 \mathrm{~dB}$ respectively at two frequencies 2.62 $\mathrm{GHz}$ and $4.57 \mathrm{GHz}$. Hence, the reflection losses parameter need to minimise and over the frequency range. The enhancement of antenna parameters are achieved by the addition of superstrate layer of Roger's 6010 having dielectric constant 10.2 and thickness $0.5 \mathrm{~mm}$.

Now by introducing the superstrate layer the modified the antenna resonates at two frequencies $2.4 \mathrm{GHz}$ and 4.20 $\mathrm{GHz}$ with an operating bandwidth of $2.56 \%$ and $2.9 \%$ respectively. Figure 5 shows the variation of return loss with the frequency for dual band implantable antenna having the superstrate layer.

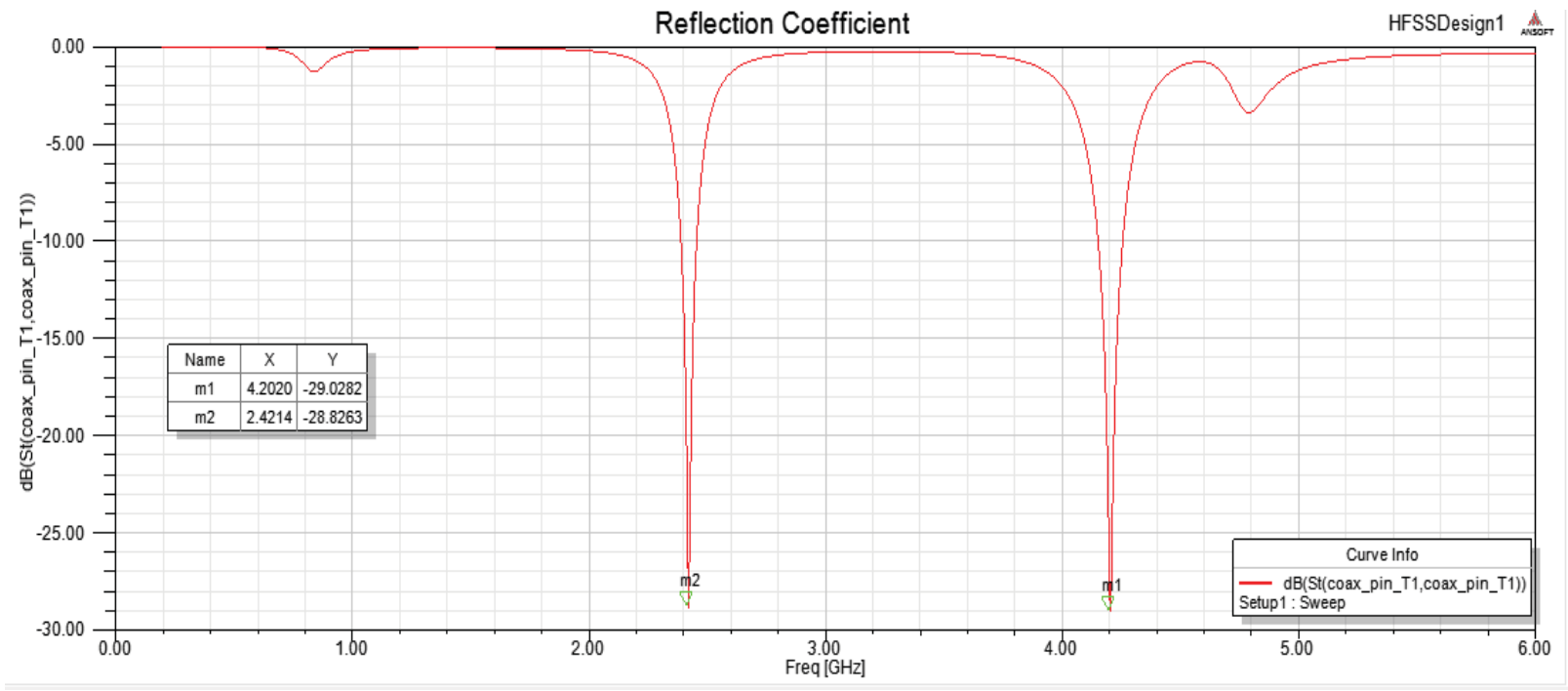

Fig. 5. Variation of return loss with frequency for implantable antenna 
The designed antenna having $\mathrm{S}_{11}$ parametre to be $-29.0282 \mathrm{~dB}$ and $-28.8263 \mathrm{~dB}$ respectively at two frequencies 2.42 $\mathrm{GHz}$ and $4.20 \mathrm{GHz}$. The simulated input impedance corresponding to two resonant frequencies is $(53.45+\mathrm{j} 1.45) \Omega$ and $(46.895-\mathrm{j} 1.445) \Omega$ respectively, as shown in Figure 6 , which is very close to 50 ohm impedance of the feed line.

\begin{tabular}{|c|c|c|c|c|}
\hline Name & $\underline{\text { Freq }}$ & $\underline{\text { Ang }}$ & $\underline{\text { Maq }}$ & $\underline{\underline{\mathrm{RX}}}$ \\
\hline $\mathrm{m} 1$ & 2.4214 & 22.0107 & 0.0362 & $1.0690+0.0290 \mathrm{i}$ \\
\hline $\mathrm{m} 2$ & 4.2020 & 154.1767 & 0.0354 & $0.9379+0.0289 \mathrm{i}$ \\
\hline
\end{tabular}
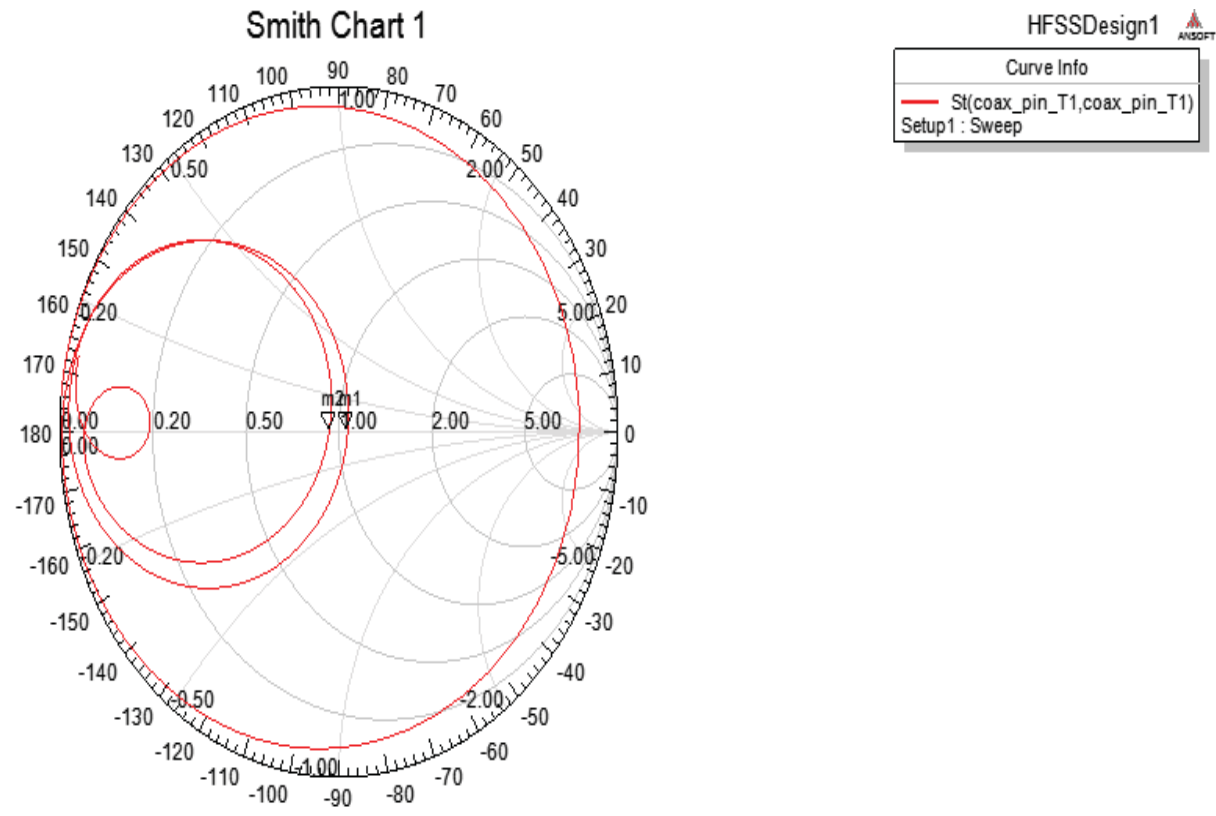

Fig. 6. Simulated input impedance of implantable antenna

It is clearly shows from figure 7 that the value of VSWR is 1.073 and 1.075 at $2.42 \mathrm{GHz}$ and $4.20 \mathrm{GHz}$ respectively. It means that the maximum power is transferred at the respective resonant frequency.

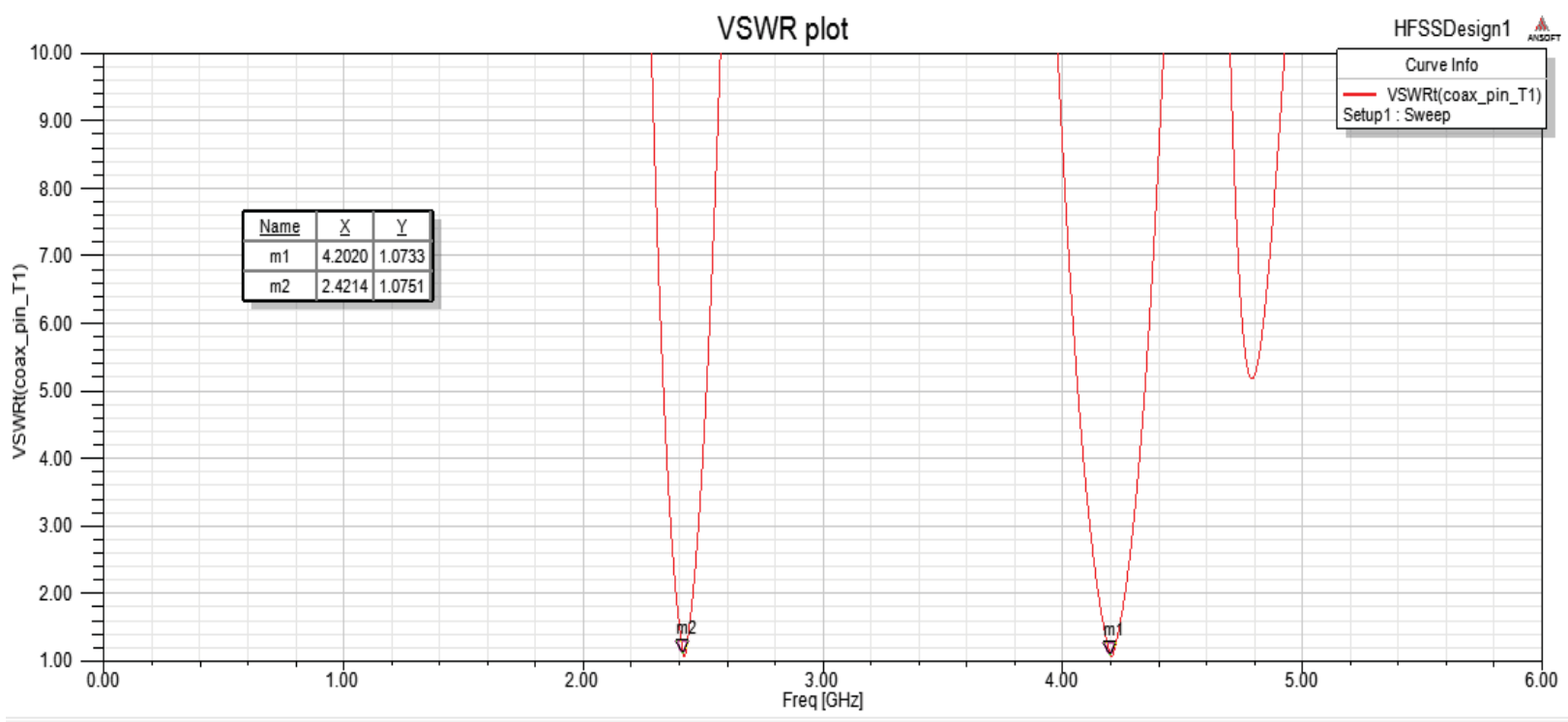

Fig. 7. Simulated VSWR of implantable antenna 
The simulated 3D radiation pattern is shown in figure 8. The simulated elevation plane radiation patterns of antenna at frequencies covering entire. It can be seen that patterns in entire bandwidth are symmetrical in nature and directed normal to the patch geometry.

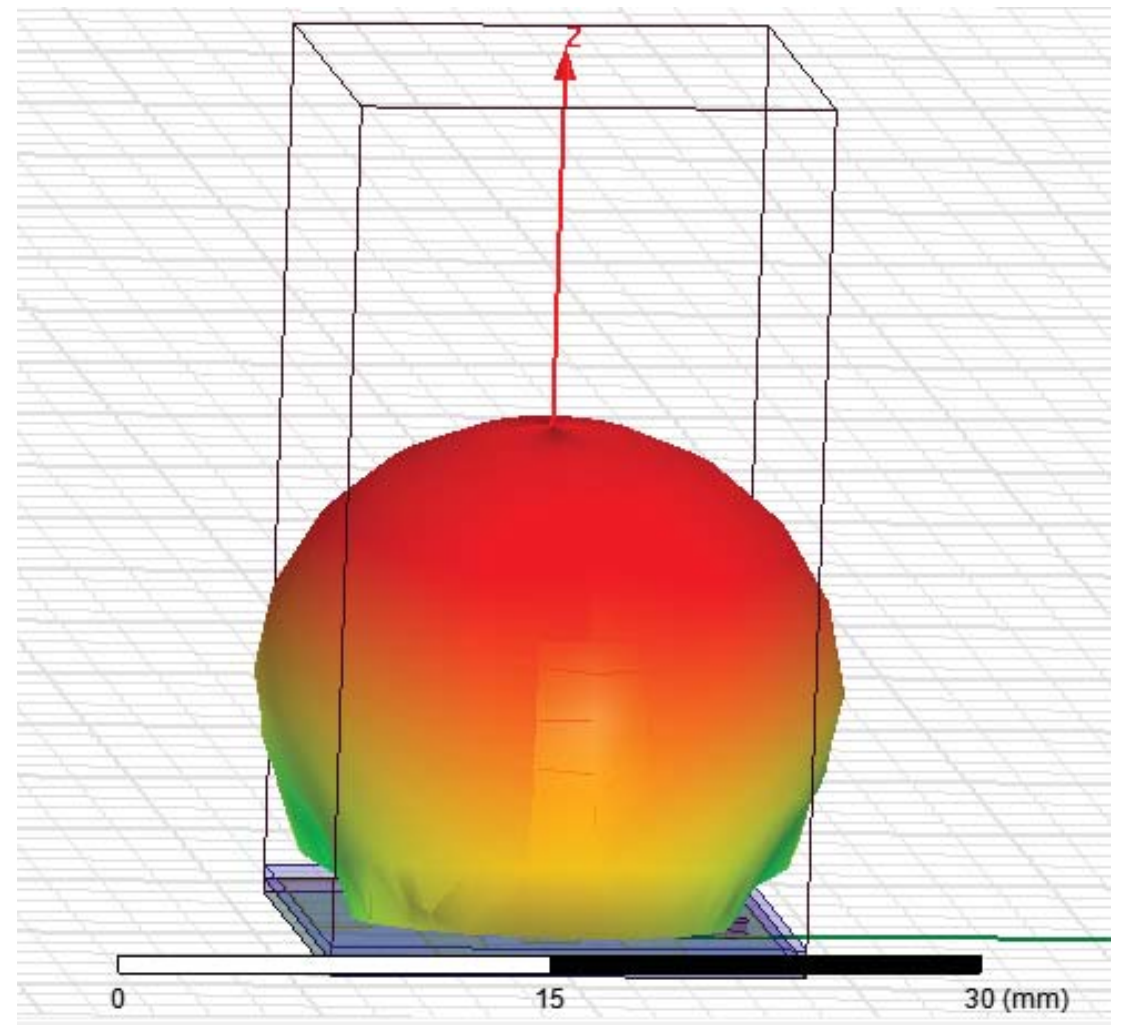

Fig. 8. Simulated 3D radiation pattern of implantable antenna

\section{CONCLUSION}

This paper presents a stacked configuration of a compact and miniature dual-band implantable antenna consists of an unsymmetrical spiral radiating stripe and a U-shaped slot is proposed for Medical Communication Services (MICS) band and Industrial, Scientific and Medical (ISM) band (2.4-2.48 GHz) applications, these are which freelicensed bands available for commercial medical use. This non-uniform spiral patch etched on the Roger's substrate having dielectric constant $\varepsilon_{\mathrm{r}}=10.2$. This simulated antenna functions on two free listened bands i.e. $2.4 \mathrm{GHz}$ to $2.48 \mathrm{GHz}$ and $4.2 \mathrm{GHz}$. The characteristics of proposed antennas have been investigated through different parametric studies using HFSS simulation software. The proposed antennas have achieved good impedance matching, stable radiation patterns, and suitable gain and bandwidth for implantable applications.

\section{ACKNOWLEDGEMENTS}

It is with great pleasure that the authors express their sincere thanks and gratitude to Dr. Abhinav Dinesh for providing simulation facilities at BIT Mesra Jaipur Campus and Dr. Deepak Bhatnagar for extending their valuable support along with the microwave laboratory at University of Rajasthan, Jaipur, India.

\section{REFERENCES}

[1] Girish Kumar and K.P.Ray, Broadband Microstrip Antennas, Artech House, Norwood, MA, 2003.

[2] K.L. Wong, Compact and Microstrip Antennas, Wiley, New York, 2003.

[3] Pozar, D.M, Microstrip Antennas, Proceeding of the IEEE, Vol.40, No.1, January 1992.

[4] S.Q. Xiao and R.Q. Li, Antennas Design for Implantable Medical Devices, School of Physical Electronics, Computational Electromagnetic (ICCEM), IEEE International Conference on University of Electronic Science and Technology of China (UESTC), 2-5 Feb. 2015, pp.61-63. 
[5] K.R. Carver and J.W. Mink, Microstrip Antenna Technology, IEEE Tran. Antennas Propagation 29, 1981, pp. 2-24.

[6] A. Kiourti and K. S. Nikita, "A review of implantable patch antennas for biomedical telemetry challenges and solutions," Vol.54, no.3, Vol. 2012, pp.210-228.

[7] P. Soontornpipit, C. M. Furse and Y. C. Chung, "Design of implantable microstrip antenna for communication with medical implants, Vol.52, no.8, Apr. 2004, pp.1944-1951.

[8] C. R. Liu, Y. X. Guo and S. Q. Xiao, "A hybrid patch/slot implantable antenna for biotelemetry devices," Antennas Wireless Propag. lett., vol.11, 2012, pp.1646-1649.

[9] Aiting Wu, Boran Guan and Zhonghai Zhang, A novel WiMAX patch antenna, IET International conference on wireless, mobile and multimedia networks, 6-9 Nov. 2006, published at IEEE Xplore, 2006, pp.1-4.

[10] A. Kiourti and K. S. Nikita, "Miniature scalp-implantable antennas for telemetry in the MICS and ISM bands: design, safety consideration and link budget analysis," Antennas Propag., Vol.60, no.8, Aug. 2012, pp. 3568-3575.

[11] Dheeraj Bhardwaj, Komal Sharma and Deepak Bhatnagar "Dual band and Broadband Rectangular Patch Mircostrip Antenna with T Shaped Slot for WiMax Application”, International Journal of Engineering Research and Development, Volume 3, Issue 12, pp. 14-21, September 2012.

[12] Dheeraj Bhardwaj, D. Bhatnagar and S. Sancheti, "Design and Development of M-shaped Dual Frequency Microstrip Antenna for Modern Communication Systems", IETE 39th Mid term Symposium on Recent advancements in Broadband Systems, April 12-13, 2008, Jaipur. 